\title{
CONTINUOUS ADDITIVE FUNCTIONALS ON THE SPACE $(B V)$ AND CERTAIN SUBSPACES
}

\author{
BY \\ C. RAYMOND ADAMS AND ANTHONY P. MORSE
}

1. Introduction. We consider here the class $(B V)$ of functions $x(t)$ of bounded variation on the interval

$$
I=E[0 \leqq t \leqq 1]
$$

Its intersection with the class $(C)$ of functions continuous on $I$ will be designated by $(C B V)$, its subclass of absolutely continuous functions by $(A C)$, and $(B V)-(C B V)$ by $(D B V)$. In a recent paper( ${ }^{(-)}$Adams introduced for $(B V)$ the metric

$$
(x, y)=\int_{0}^{1}|x(t)-y(t)| d t+\left|T_{0}^{1}(x)-T_{0}^{1}(y)\right|,
$$

$T_{0}^{1}(z)$ being employed in general to denote the total variation of the function $z(t)$ on $I$. Thus metrised, $(B V)$ is not a Banach space $\left({ }^{2}\right)$; but it is complete, separable, and boundedly compact. Although a linear space, it is not a "linear topological space" in the sense in which that term is sometimes used, for the topology introduced by the metric (1) is non-uniform. Indeed it is easily seen that the category of a subset is not always invariant under translation. For, if the closed unit sphere $K(\theta, 1)$ about the zero-element $\theta$ as center, which is a set of second category in $(B V)$, is subjected to the translation $x$ where $x$ has as a representative function $x(t)=0$ for $0<t \leqq 1, x(0)=2$, then its translate

$$
E=\underset{y}{E}[y=x+z, z \varepsilon K]
$$

is a subset of $(D B V)$ and so of first category $\left({ }^{3}\right)$ in $(B V)$. Regarded as a group, $(B V)$ is discontinuous.

Presented to the Society, December 2, 1939; received by the editors January 25, $1940^{\circ}$

(') Adams, The space of functions of bounded variation and certain general spaces, these Transactions, vol. 40 (1936), pp. 421-438, hereinafter referred to as A. The properties of ( $B V)$ mentioned presently are either explicitly established in, or easily to be inferred from the results of, this paper and its sequel by Adams and Morse, On the space ( $B V)$, ibid., vol. 42 (1937), pp. 194-205, later referred to as AM.

(2) Indeed it is clear, from the fact that "convergence in variation" is not additive, that it is impossible to norm the set $(B V)$, or either of its subsets $(C B V)$ and $(A C)$, in such manner that convergence in the metric determined by the norm is equivalent to convergence in the metric (1). See Adams and Clarkson, On convergence in variation, Bulletin of the American Mathematical Society, vol. 40 (1934), pp. 413-417. This same remark holds for $(B V)$ or $(C B V)$ metrised with the distance function (17); see $\S 6$ below.

${ }^{(3)}$ See AM, p. 199. An example of a residual set which under the same translation goes into a set of first category is provided by $(C B V)$. 
2. Functionals on $(B V)$. The functional $\left({ }^{4}\right) f(x)=\operatorname{ess~} \lim _{t \rightarrow 0} x(t)=\lim _{t \rightarrow 0} x(t)$ clearly is defined for every $x \varepsilon(B V)$ in the natural sense that if $x(t)$ is an element of the class $(B V), f(x)$ is a real number, and if $x(t)$ and $y(t)$ are both elements of the class $(B V),(x, y)=0$ implies $f(x)=f(y)$. This functional is additive and homogeneous on $(B V)$, and it may readily be seen to be continuous at each point of the subset $(B V N)$ corresponding to functions having no external saltus anywhere $\left(^{5}\right)$; nevertheless it is discontinuous at each point of $(B V)-(B V N)$. Incidentally, both $(B V N)$ and its complement are dense in $(B V)$. In further contrast to the situation in the case of a Banach space, there exist functionals which are additive and continuous on $(B V)$ without being uniformly continuous. But any functional $f(x)$ which is additive and uniformly continuous on $(B V)$ does satisfy a Lipschitz condition, $|f(x)-f(y)| \leqq M \cdot(x, y)$ for $x, y \varepsilon(B V)$; and the smallest number $M$ which can be used in this inequality we have called the "modulus" of $f$ on $(B V)$ and designated by the symbol $\bmod _{(B V)} f$.

In $\mathrm{A}$, Theorems 5.1, 5.2, and 5.3, it was shown that every functional $f$ additive and uniformly continuous on $(B V)$ [or on $(C B V)$ or on $(A C)]$ can be expressed in the form of a Lebesgue integral,

$$
\int_{0}^{1} x(t) \alpha(t) d t, \quad \operatorname{ess}_{\sup _{t \varepsilon I}}|\alpha(t)|=M<\infty,
$$

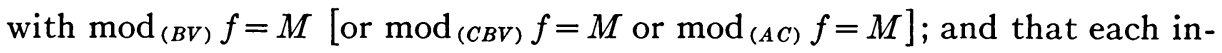
tegral of this kind is such a functional $\left({ }^{6}\right)$. An example of an additive and continuous functional which is not uniformly continuous on $(B V)$ is provided by any such integral with $\alpha(t)$ summable but not essentially bounded. The general form of the additive and continuous, but not necessarily uniformly continuous, functional on $(B V)$, however, was not determined in $\mathrm{A}$. This open question we now propose to settle.

THEOREM 1. The conditions $T_{0}^{1}\left(x_{n}\right)<B<\infty \quad(n=0,1,2, \cdots), \lim _{n \rightarrow \infty}$ $\int_{0}^{1}\left|x_{n}-x_{0}\right| d t=0$, and $g \boldsymbol{\varepsilon}(C)$ imply $\lim _{n \rightarrow \infty} \int_{0}^{1} x_{n} d g=\int_{0}^{1} x_{0} d g$.

Proof. This theorem is equivalent to Bray's extension $\left({ }^{7}\right)$ of a theorem of

(4) By functional we mean an operation or transformation whose range is contained in the real number system. We recall the well known fact that in a Banach space continuity of an additive functional $f$ at one point alone implies continuity everywhere, uniform continuity, and the satisfaction by $f$ of a Lipschitz condition on the entire space.

$\left.{ }^{5}\right)$ For a precise definition of $(B V N)$ see the first paragraph of $\$ 3$ below.

$\left.{ }^{(}\right)$More recently Hildebrandt, in Linear operations on functions of bounded variation, Bulletin of the American Mathematical Society, vol. 44 (1938), p. 75, has determined the general form of the continuous additive functional on the non-separable Banach space which the class ( $B V)$ becomes when normed with $\|x\|=|x(0)|+T_{0}^{1}(x)$. As in the case of other non-separable Banach spaces previously considered by this author, the functional is expressed by a generalized integral of Stieltjes or Lebesgue type which he constructs for the purpose.

(7) See Bray, Elementary properties of the Stieltjes integral, Annals of Mathematics, (2), vol. 20 (1918-1919), p. 180. 
Helly, in the sense that each can be derived from the other. It seems preferable to us, however, to prove our result di novo rather than to use Bray's theorem as a basis. That the first two conditions imply uniform boundedness of $x_{n}$, and that all three imply $\lim _{n \rightarrow \infty} \int_{0}^{1} x_{n} d g=\int_{0}^{1} x_{0} d g$ in the particular case in which $g \varepsilon(A C)$, has already been remarked in the first paragraph of the proof of Theorem 5.1 of A. We now extend the proof to the general case, in which $g$ is an arbitrary continuous function. Let $\epsilon$ be any positive number; let $B_{1} \geqq B$ be a bound for $\left|x_{n}(t)-x_{0}(t)\right|(t \varepsilon I ; n=1,2,3, \cdots)$; and let $h(t)$ satisfy the conditions

$$
h \varepsilon(A C), \sup _{t \varepsilon I}|g(t)-h(t)| \leqq \epsilon /\left(2 B_{1}\right), \quad g(0)-h(0)=g(1)-h(1)=0 .
$$

In accordance with the particular case of the theorem already proved we have $\lim _{n \rightarrow \infty} \int_{0}^{1}\left(x_{n}-x_{0}\right) d h=0$, whence as $n \rightarrow \infty$

$$
\begin{aligned}
\lim \sup \left|\int_{0}^{1}\left(x_{n}-x_{0}\right) d g\right| \leqq & \lim \sup \left|\int_{0}^{1}\left(x_{n}-x_{0}\right) d(g-h)\right| \\
& +\lim \sup \left|\int_{0}^{1}\left(x_{n}-x_{0}\right) d h\right| \\
= & \lim \sup \left|\int_{0}^{1}(g-h) d\left(x_{n}-x_{0}\right)\right| \\
\leqq & \lim \sup \frac{\epsilon}{2 B_{1}} T_{0}^{1}\left(x_{n}-x_{0}\right) \\
& \leqq \\
& \lim \sup \frac{\epsilon}{2 B_{1}}\left[T_{0}^{1}\left(x_{n}\right)+T_{0}^{1}\left(x_{0}\right)\right] \leqq \epsilon .
\end{aligned}
$$

Corollary. Each integral

$$
\int_{0}^{1} x(t) d g(t)
$$

with $g \boldsymbol{\varepsilon}(C)$

is a continuous additive functional for $x \varepsilon(B V)$.

THEOREM 2. Each continuous additive functional on $(B V)$ can be expressed in the form (3).

Proof. Let $f$ be any such functional and, as in the proof of Theorem 5.2 of $\mathrm{A}$, set

$$
\xi_{t}(u)=\left\{\begin{array}{l}
1 \text { for } 0 \leqq u \leqq t, \\
0 \text { for } t<u \leqq 1,
\end{array} \quad f\left(\xi_{t}\right)=g(t), \quad t \in I .\right.
$$

For any pair of numbers $t, t_{1}$ in the interval $0 \leqq t<1$ we have $\left(\xi_{t}, \xi_{t_{1}}\right)$ $=\int_{0}^{1}\left|\xi_{t}-\xi_{t_{1}}\right| d u$, so that $t \rightarrow t_{1}$ implies $\left(\xi_{t}, \xi_{t_{1}}\right) \rightarrow 0$. This in turn implies 
$f\left(\xi_{t}\right) \rightarrow f\left(\xi_{t_{1}}\right)$, since $f$ is continuous at $\xi_{t_{1}}$; i.e., $t \rightarrow t_{1}$ implies $g(t) \rightarrow g\left(t_{1}\right)$. If we let $\eta(u)=1$ for $0 \leqq u<1, \eta(1)=0$, it is clear that $t \rightarrow 1$ implies $\left(\xi_{t}, \eta\right)=\int_{0}^{1}\left|\xi_{t}-\eta\right| d u$ $\rightarrow 0$, which implies $f\left(\xi_{t}\right) \rightarrow f(\eta)$; i.e., we may infer that $\lim _{t \rightarrow 1} g(t)$ exists. Hence, defining $\bar{g}(t)=g(t)$ for $0 \leqq t<1, \bar{g}(1)=\lim _{t \rightarrow 1} g(t)$, we have $\bar{g} \varepsilon(C)$ and $\int_{0}^{1} x d \bar{g}$ exists for every $x \varepsilon(B V)$. The argument used in the proof of Theorem 5.2 of $\mathrm{A}$ now holds, with no change whatever, from the beginning of the second paragraph up to and including equation (5.4); that is to say, it may be concluded that $g(1)=\bar{g}(1)$ and $f(x)=\int_{0}^{1} x(t) d g(t)$ for every $x \varepsilon(B V)$.

3. Functionals on $(C B V)$ and $(A C)$. An arbitrary function $x$ will be said to have no external saltus if and only if at each point $t_{1} \varepsilon I, x$ satisfies the condition $\lim \inf _{t \rightarrow t_{1}} x(t) \leqq x\left(t_{1}\right) \leqq \lim \sup _{t \rightarrow t_{1}} x(t)$. We shall employ $(B V N)$ to designate the intersection of the class $(B V)$ with the class of functions having no external saltus. Clearly $x \varepsilon(B V N)$ implies continuity of $x$ at $t=0$ and $t=1$.

Theorem 3. The conditions $\left({ }^{8}\right) x_{n} \varepsilon(B V)(n=1,2,3, \cdots), x_{0} \varepsilon(B V N)$, $\lim _{n \rightarrow \infty}\left(x_{n}, x_{0}\right)=0,|g(t)|<B<\infty$ fort $\varepsilon I$, and $\int_{0}^{1} g d x_{n} \operatorname{exists}\left({ }^{9}\right)(n=0,1,2, \cdots)$ imply $\lim _{n \rightarrow \infty} \int_{0}^{1} g d x_{n}=\int_{0}^{1} g d x_{0}$ and $\lim _{n \rightarrow \infty} \int_{0}^{1} x_{n} d g=\int_{0}^{1} x_{0} d g$.

Proof. In the same manner in which it may be seen that a closed set $E \subset I$ can be inclosed in a finite set of disjoint intervals $O_{i}$ each open with respect to $I$ and the sum of whose lengths exceeds the measure of $E$ by arbitrarily little, one may see that $E$ can be inclosed in a finite set of such intervals with $\sum_{i} T_{\bar{o}_{i}}\left(x_{0}\right)$ exceeding the variation $\left({ }^{9}\right)$ of $x_{0}$ on $E$ by an arbitrarily small amount.

Let $\epsilon$ be an arbitrary positive number, $k$ a positive number satisfying the inequality $k T_{0}^{1}\left(x_{0}\right)<\epsilon$, and $D_{k} \subset I$ the set of points where $g$ has a saltus $\geqq k$. Since $D_{k}$ is closed and the variation of $x_{0}$ on $D_{k}$ is zero, $D_{k}$ can be inclosed in a finite set of disjoint intervals $O_{i}$, each open with respect to $I$, such that $\sum_{i} T_{\bar{o}_{i}}\left(x_{0}\right)<\epsilon /(2 B)$. Since the points of continuity of $x_{0}$ are dense in $I$ and include 0 and 1 , and since $D_{k}$ is closed, each interval $O_{i}$ can be shrunk (if necessary) into an interval $O_{i}^{\prime}$, open with respect to $I$, whose end-points are points of continuity of $x_{0}$ and such that $D_{k}$ is still inclosed in $\sum_{i} O_{i}^{\prime}$. By

( $\left.{ }^{8}\right)$ From the proof it will be clear that a weaker set of conditions sufficient to insure the conclusion is obtained by replacing $\left(x_{n}, x_{0}\right) \rightarrow 0$ by the following: $\int_{0}^{1}\left|x_{n}-x_{0}\right| d t \rightarrow 0$ and the existence of a set $D$ dense in $I$ and containing 0 and 1 and of a non-decreasing function $F$ such that $\int_{0}^{1} g d F$ exists and on every closed interval $J \subset I$ with end-points in $D, \lim \sup _{n \rightarrow \infty} T_{J}\left(x_{n}\right)$ does not exceed the increment of $F$ on $J$. We take occasion to remark that this theorem neither includes nor is included by a theorem of Daniell on passage to the limit, Further properties of the general integral, Annals of Mathematics, (2), vol. 21 (1919-1920), p. 218.

$\left({ }^{9}\right)$ It is desirable to recall here the meaning of the term "variation of a function on a set." Let $x \varepsilon(B V)$ and $E \subset I$; then the variation of $x$ on $E$ is by definition the infimum of numbers of the form $\sum_{i} T_{o_{i}}(x)$ where $E \subset \sum_{i} O_{i}$, each $O_{i}$ is an interval open with respect to $I$ (i.e., $O_{i}$ is the intersection with $I$ of some open interval), and $\bar{O}_{i}$ is the closure of $O_{i}$. If $g$ is bounded on $I$ and $x \varepsilon(B V)$, a necessary and sufficient condition for $\int_{0}^{1} g d x$ to exist is that the variation of $x$ on the set of points of discontinuity of $g$ be zero. See, for example, Hobson, Theory of Functions of a Real Variable, 3d edition, vol. 1, Cambridge, 1927, p. 542. 
Theorem 1 of AM, $\left(x_{n}, x_{0}\right) \rightarrow 0$ implies the same condition on each subinterval $\bar{O}_{i}^{\prime}$. Letting $\alpha=\sum_{i} \bar{O}_{i}^{\prime}, T_{\alpha}\left(x_{n}\right)=\sum_{i} T_{\bar{O}_{i}^{\prime}}^{\prime}\left(x_{n}\right)(n=0,1,2, \cdots)$, we therefore have as $n \rightarrow \infty$

$$
\begin{aligned}
\lim \sup \left|\int_{\alpha} g d x_{n}-\int_{\alpha} g d x_{0}\right| & \leqq \lim \sup \left|\int_{\alpha} g d x_{n}\right|+\lim \sup \left|\int_{\alpha} g d x_{0}\right| \\
& \leqq \lim \sup B \cdot\left[T_{\alpha}\left(x_{n}\right)+T_{\alpha}\left(x_{0}\right)\right] \\
& =2 B T_{\alpha}\left(x_{0}\right)<\epsilon .
\end{aligned}
$$

Let $\beta$ denote the finite set of disjoint closed intervals constituting the closure of the point set $I-\alpha$, at no point of which $g$ has a saltus $\geqq k$. By aid of the Heine-Borel theorem $\left({ }^{10}\right)$ one may easily see that on each interval of $\beta, g$ can be approximated uniformly within $k / 2$ by a continuous function $h$. We then have as $n \rightarrow \infty$

$$
\begin{aligned}
\lim & \sup \left|\int_{\beta} g d x_{n}-\int_{\beta} g d x_{0}\right| \\
& =\lim \sup \left|\int_{\beta}(g-h) d x_{n}+\int_{\beta} h d x_{n}-\int_{\beta}(g-h) d x_{0}-\int_{\beta} h d x_{0}\right| \\
& \leqq \lim \sup \left|\int_{\beta}(g-h) d x_{n}-\int_{\beta}(g-h) d x_{0}\right|+\lim \sup \left|\int_{\beta} h d x_{n}-\int_{\beta} h d x_{0}\right| \\
& \leqq \lim \sup (k / 2)\left[T_{\beta}\left(x_{n}\right)+T_{\beta}\left(x_{0}\right)\right]=k T_{\beta}\left(x_{0}\right)<\epsilon,
\end{aligned}
$$

since $\lim _{n \rightarrow \infty} \int_{\beta} h d x_{n}=\int_{\beta} h d x_{0}$ is an immediate consequence of Theorem 1 , the formula for integration by parts, and the fact that by Theorem 2 of AM we have $\left.{ }^{11}\right)$ pointwise convergence of $x_{n}(t)$ to $x_{0}(t)$ at each end-point of the intervals constituting $\beta$. That $\int_{0}^{1} x_{n} d g \rightarrow \int_{0}^{1} x_{0} d g$ now follows at once by aid of the formula for integration by parts and the pointwise convergence of $x_{n}(t)$ to $x_{0}(t)$ at $t=0$ and $t=1$.

(10) An explicit construction for $h$, on an interval which may as well be taken as $I$, is the following. Let $M(t), N(t)$ be the "maximum and minimum functions" for $g$ (i.e., for example, let $M(t)=\lim _{\Delta \rightarrow 0} \sup _{t_{1} \varepsilon} \varepsilon,\left|t_{1}-t\right|<\Delta g\left(t_{1}\right)$ for $\left.t \varepsilon I\right)$; the saltus of $g$ at $t$ is then $M(t)-N(t)$, and this is $<k$ for $t \varepsilon I$. Setting $M_{n}(t)=\sup _{t_{1} \varepsilon}\left[f\left(t_{1}\right)-n\left|t_{1}-t\right|\right], N_{n}(t)=\inf _{t \varepsilon}\left[f\left(t_{1}\right)+n\left|t_{1}-t\right|\right]$ $(t \varepsilon I ; n=1,2,3, \cdots)$, we easily see that for each $n$ these functions satisfy a Lipschitz condition, and that $M_{n}(t) \rightarrow M(t)$ from above and $N_{n}(t) \rightarrow N(t)$ from below as $n \rightarrow \infty$, so that $M_{n}(t)-N_{n}(t)$ tends to $M(t)-N(t)$ from above. Let

$$
E_{n}=E_{\iota}\left[M_{n}(t)-N_{n}(t) \geqq k\right] ;
$$

then each $E_{n}$ is closed, each $E_{n} \supset E_{n+1}$, and $\prod_{n=1}^{\infty} E_{n}$ is vacuous. Hence there exists an integer $n_{0}$ for which $E_{n_{0}}$ is vacuous; i.e., we have $M_{n_{0}}(t)-N_{n_{0}}(t)<k$ for $t \varepsilon I$. The desired function $h$ may now be taken to be $\left[M_{n_{0}}(t)+N_{n_{0}}(t)\right] / 2$ for $t \varepsilon I$.

(11) According to this theorem the conditions $x_{n} \varepsilon(B V)(n=1,2,3, \cdots), x_{0} \varepsilon(B V N)$, and $\left(x_{n}, x_{0}\right) \rightarrow 0$ imply pointwise convergence of $x_{n}$ to $x_{0}$ at each point of continuity of $x_{0}$. 
For convenience in stating the following corollaries we let $(R)$ stand for the class of functions which are Riemann integrable on $I$ and $\left(R^{*}\right)$ for the subclass of $(R)$ of which each function has only a countable number of discontinuities. Recalling $\left({ }^{12}\right)$ that each pair of conditions, $x \varepsilon(C B V), g \varepsilon\left(R^{*}\right)$ and $x \varepsilon(A C), g \varepsilon(R)$, is sufficient to insure the existence of $\int_{0}^{1} x d g$, we have

Corollary 1. Each integral

$$
\int_{0}^{1} x(t) d g(t)
$$
with $g \varepsilon\left(R^{*}\right)$

is a continuous additive functional for $x \boldsymbol{\varepsilon}(C B V)$.

Corollary 2. Each integral

$$
\int_{0}^{1} x(t) d g(t)
$$

with $g \varepsilon(R)$

is a continuous additive functional for $x \boldsymbol{\varepsilon}(A C)$.

In conjunction with Theorem 2, Corollary 1 shows that there exist continuous additive functionals on $(C B V)$ which cannot be extended to be continuous and additive on $(B V)$. In determining the general form of such a functional on $(C B V)$ it is therefore desirable, if not actually necessary, to work wholly within the space $(C B V)$ itself. We propose to prove that the general form of such a functional on $(C B V)$ is $(4)$ and on $(A C)$ is (5). For this purpose we shall employ two lemmas as follows. Allowing $(A C)_{0}$ to denote the subset of $(A C)$ of which each function vanishes at $t=0$, we have

LEMмa 1. Let $f$ be a functional whose domain includes $(A C)_{0}$. If $f$ is additive on $(A C)_{0}$ and continuous on $(A C)_{0}$, metrised with the distance function $(x, y)=T_{0}^{1}(x-y)$, there exists a function h bounded and summable on I such that

$$
f(x)=\int_{0}^{1} h(t) x^{\prime}(t) d t \quad \text { for } x \varepsilon(A C)_{0} .
$$

This result is an immediate consequence of the facts that $(A C)_{0}$ is isometric with the Banach space $(L)$ of functions summable on $I$ and that for $x^{\prime} \varepsilon(L)$ the general form of the continuous additive functional $f\left(x^{\prime}\right)$ on $(L)$ is given $\left({ }^{13}\right)$ by the integral in (6). Since convergence in the metric $(x, y)=T_{0}^{1}(x-y)$ implies convergence in the metric (1), any functional $f$ whose domain includes $(A C)_{0}$ and which is additive and continuous on $(A C)_{0}$ metrised with (1) can be expressed, for $x \varepsilon(A C)_{0}$, in the form (6).

To promote our later convenience we formulate the

(12) See, for example, Hobson, loc. cit., p. 545.

(13) See, for example, Banach, Théorie des Opêrations Linéaires, Warsaw, 1932, p. 65. 
Definition. The function $h$ in (6) shall be called the normalized function associated with the functional $f$ if and only if it has the properties

$$
\begin{aligned}
& h(0)=f\left(x_{1}\right) \text { where } x_{1}(t)=1 \text { for } t \varepsilon I, \quad h(1)=0, \\
& h(t)=\frac{1}{2}\left[\lim \sup _{\delta \rightarrow 0} \int_{t}^{t+\delta} h(u) d u / \delta+\lim \inf _{\delta \rightarrow 0} \int_{t}^{t+\delta} h(u) d u / \delta\right] \\
& \quad \text { for } 0<t<1 .
\end{aligned}
$$

It should be clear from Lemma 1 and from this definition that associated with each functional $f$ of the kind specified there is a normalized function $h$; and that if $\epsilon$ is an arbitrary positive number and $t_{1}$ an arbitrary point in the open interval $0<t<1$, there exists in every neighborhood of $t_{1}$ a set of positive measure on which $h(t)$ is $<h\left(t_{1}\right)+\epsilon$ and a set of positive measure on which $h(t)$ is $>h\left(t_{1}\right)-\epsilon$.

It follows at once that any functional $f$ whose domain includes $(A C)$, and which is additive and continuous on $(A C)$ metrised with the distance function $(x, y)=|x(0)-y(0)|+T_{0}^{1}(x-y)$, can be expressed in the form $\left({ }^{14}\right)$

(8) $f(x)=x(0) f\left(x_{1}\right)+\int_{0}^{1} h(t) x^{\prime}(t) d t=x(0) h(0)+\int_{0}^{1} h(t) x^{\prime}(t) d t$ for $x \varepsilon(A C)$,

where $h$ has the properties ( 7$)$.

LeMmA 2. Let $\delta$ be an arbitrary number $>0 ; P$ a non-vacuous perfect set $\subset I ; x(t)$ a continuous non-decreasing function $\left({ }^{15}\right)$ with $x(0)=0, x(1)=1$, and $x^{\prime}(t)=0$ for $t \varepsilon I-P$; and $h(t)$ a bounded measurable function for $t \varepsilon I$, with essential saltus $\geqq k>0$ at each point of $P$. Then there exist two non-decreasing functions $\lambda(t), \mu(t) \varepsilon(A C)_{0}$ satisfying the conditions

$$
\begin{gathered}
\sup _{t \varepsilon I}|\lambda(t)-x(t)|<\delta, \quad \sup _{t \varepsilon I}|\mu(t)-x(t)|<\delta, \\
\int_{0}^{1} h(t) \lambda^{\prime}(t) d t-\int_{0}^{1} h(t) \mu^{\prime}(t) d t \geqq k / 2 .
\end{gathered}
$$

(14) The usual scheme for determining the general form of the continuous additive functional $f$ on the Banach space $(C)$ employs the device of extending $f$ to points outside of $(C)$; i.e., to the class of step-functions or to the entire space $(M)$ of essentially bounded measurable functions. It may therefore be of interest to note that we now have in hand a means of obtaining the form of $f$ without going outside of $(C)$ itself. In fact from (8), by following essentially the same procedure as is used below in the proof of Lemma 3 (only that in the present instance $x_{1}$ should be taken as $x_{1}(t)=0$ for $t \varepsilon I, \epsilon$ should be taken as 1 , and we have no concern with the values of $\int_{0}^{1}\left|x_{\delta, \epsilon}(t)\right| d t$ and $\left.L_{0}^{1}\left(x_{\delta, \epsilon}\right)\right)$, one may readily show $\sup _{x} \varepsilon(A C),\|x\|=1 f(x) \geqq T_{0}^{1}(h)$. Letting $g(t)=h(0)-h(t)$ for $t \varepsilon I$, and observing that $(A C)$ is dense in $(C)$, we may conclude $f(x)=\int_{0}^{1} x d g$ for $x \varepsilon(C)$ and $\|f\|_{(C)}=T_{0}^{1}(g)=T_{0}^{1}(h)$.

(15) One readily sees that, for any non-vacuous $P$, there exists a function $x$ on $I$ with these properties; for example, in any subinterval of $I$ where $P$ is dense, $x(t)$ may be taken as $t$, and elsewhere it may be defined by essentially the same process as is used for defining the Cantor ternary function. 
Proof. Let $N$ be a positive integer large enough so that

$$
\left|x\left(t_{1}\right)-x\left(t_{2}\right)\right|<\delta \quad \text { for }\left|t_{1}-t_{2}\right|<1 / N,
$$

and let $I_{n}(n=1,2, \cdots, N)$ stand for the interval $(n-1) / N<t<n / N$. For each $n$ we define functions $\lambda_{n}, \mu_{n}$ as follows. If $P \cdot I_{n}$ is vacuous, set $\lambda_{n}(t)=\mu_{n}(t)$ $=0$ for $t \varepsilon I$. If $P \cdot I_{n}$ is non-vacuous, set

$$
S_{n}=\operatorname{ess}_{\sup _{i \varepsilon I_{n}}} h(t), \quad s_{n}=\operatorname{ess}_{\inf _{t \varepsilon I_{n}}} h(t),
$$

so that $S_{n}-s_{n} \geqq k$; let $\alpha_{n}, \beta_{n}$ be any measurable subsets of $I_{n}$ with

$$
\begin{array}{ll}
\left|\alpha_{n}\right|>0, & \left|S_{n}-h(t)\right|<k / 4 \text { for } t \varepsilon \alpha_{n}, \\
\left|\beta_{n}\right|>0, & \left|s_{n}-h(t)\right|<k / 4 \text { for } t \varepsilon \beta_{n} ;
\end{array}
$$

and set

$$
\lambda_{n}(t)=\left\{\begin{array}{ll}
\Delta_{I_{n}} x /\left|\alpha_{n}\right| & \text { for } t \varepsilon \alpha_{n}, \\
0 & \text { for } t \varepsilon I-\alpha_{n},
\end{array} \quad \mu_{n}(t)= \begin{cases}\Delta_{I_{n}} x /\left|\beta_{n}\right| & \text { for } t \varepsilon \beta_{n}, \\
0 & \text { for } t \varepsilon I-\beta_{n},\end{cases}\right.
$$

where the notation $|E|$ stands for the measure of a set $E$ and $\Delta_{I_{n}} x$ represents the increment of the function $x$ on the closure of the interval $I_{n}$. We observe

$$
\begin{aligned}
\int_{0}^{1} h(t) \lambda_{n}(t) d t & =\int_{\alpha_{n}} h(t) \lambda_{n}(t) d t \\
& \geqq \int_{\alpha_{n}}\left(S_{n}-k / 4\right) \lambda_{n}(t) d t=(S-k / 4) \Delta_{I_{n}} x, \\
\int_{0}^{1} h(t) \mu_{n}(t) d t & \leqq\left(s_{n}+k / 4\right) \Delta_{I_{n}} x,
\end{aligned}
$$

and we assert that the non-decreasing functions

$$
\lambda(t)=\int_{0}^{t} \sum_{n=1}^{N} \lambda_{n}(u) d u, \quad \mu(t)=\int_{0}^{t} \sum_{n=1}^{N} \mu_{n}(u) d u
$$

have the properties (9). It is clear that $\lambda(t)=\mu(t)=x(t)$ for $t=n / N$ $(n=0,1, \cdots, N)$, whence in view of $(10)$ the first two of inequalities (9) are satisfied. As for the third, we have by (11)

$$
\begin{aligned}
\int_{0}^{1} h(t)\left[\lambda^{\prime}(t)-\mu^{\prime}(t)\right] d t=\int_{0}^{1} h(t) \sum_{n=1}^{N}\left[\lambda_{n}(t)-\mu_{n}(t)\right] d t \\
=\sum_{n=1}^{N} \int_{0}^{1} h(t) \lambda_{n}(t) d t-\sum_{n=1}^{N} \int_{0}^{1} h(t) \mu_{n}(t) d t \\
\geqq \sum_{n=1}^{N}\left(S_{n}-k / 4-s_{n}-k / 4\right) \Delta_{I_{n}} x \geqq(k / 2) \sum_{n=1}^{N} \Delta_{I_{n}} x=k / 2 .
\end{aligned}
$$


THEOREM 4. Each continuous additive functional on $(C B V)$ can be expressed in the form (4).

Proof. Each functional $f(x)$ of this kind is expressible, for $x \varepsilon(A C)$, in the form (8). The function $h$ can have an essential discontinuity at no more than a countable set of points; for the contrary would imply the existence of a number $k>0$ such that the points where $h$ has an essential saltus $\geqq k$ would be a non-countable closed set, this set would contain a non-vacuous perfect set, and by Lemma 2 there would exist points $x \varepsilon(C B V)$ at which $f$ fails to be continuous. Being normalized, $h$ is continuous whenever it is essentially continuous; hence $h$ is continuous except at a countable set of points and we may write

$$
x(0) h(0)+\int_{0}^{1} h(t) x^{\prime}(t) d t=x(0) h(0)+\int_{0}^{1} h(t) d x(t) .
$$

This expression may be brought in to the form (4) by setting $g(t)=h(0)-h(t)$.

THEOREM 5. Each continuous additive functional on $(A C)$ can be expressed in the form (5).

This result can be demonstrated in the same manner as Theorem 4 , the conclusion that $h$ cannot have essential discontinuities at a set $D$ of measure $>0$ being drawn from Lemma 2. For, if $|D|$ were $>0$, there would exist a $k>0$ such that the set $D_{k} \subset D$ where $h$ has an essential saltus $\geqq k$ would be closed and of measure $>0$; and $D_{k}$ would contain a perfect set $P$ with $|P|>0$. The function $x$ of Lemma 2 could then be taken as $\int_{0}^{t} \phi(u) d u /|P|$, where $\phi$ is the characteristic function of $P \subset I$; i.e., there would exist points $x \varepsilon(A C)_{0}$ at which $f$ fails to be continuous.

4. Norms of the functionals. By Theorem 2.3 of A, any functional $f$ additive and continuous on $(B V)$ satisfies a Lipschitz condition at the zeroelement $\theta \varepsilon(B V)$, with a Lipschitz modulus which was called the "norm" of $f$ on $(B V)$ and designated by the symbol $\|f\|_{(B V)}$. On the assumption that $g(0)=0$, which can be made without loss of generality, the upper bounds

$$
T_{0}^{1}(g), \quad|g(1)|+\sup _{t \varepsilon I}|g(t)|
$$

and the lower bounds

$$
\frac{1}{3} \operatorname{osc}_{t \varepsilon} I g(t), \quad|g(1)|, \quad \frac{1}{2} \sup _{t \varepsilon I}|g(t)|
$$

for $\|f\|_{(B V)}$ were determined $\left({ }^{16}\right)$ in A. These show that if $g$ is monotone on $I$, $\|f\|_{(B V)}=T_{0}^{1}(g)$; in no other case, however, was the norm evaluated. We now propose to evaluate the norms of the functionals (3), (4), and (5).

${ }^{(16)}$ See A, pp. 437-438. It was tacitly assumed there that the functional $f$ under consideration was uniformly continuous on $(B V)$; but this assumption was not used, the bounds being determined solely from the Stieltjes integral form of the functional. 
For convenience in this connection we adopt the following conventions. If $g$ is a function on $I$ and

$$
J=\underset{t}{E}\left[t^{\prime} \leqq t \leqq t^{\prime \prime}\right] \subset I
$$

we define $\Delta_{J}^{\prime} g=g\left(t^{\prime \prime}\right)-g\left(t^{\prime}\right)$ when each of the points $t^{\prime}, t^{\prime \prime}$ is an end-point of $I$ or a point of continuity of $g, \Delta_{J}^{\prime} g=0$ otherwise; $|J|$ will stand for the length of $J$; and $\nu(J)$ will be 0,1 , or 2 according as both, one, or neither of the conditions $0 \varepsilon J, 1 \varepsilon J$ is satisfied. We then have

THEOREM 6. The norm of each of the functionals (3), (4), and (5) is

$$
\sup _{J}\left|\Delta_{J}^{\prime} g\right| /[|J|+\nu(J)]
$$

as $J$ ranges over the set of closed subintervals of $I$.

Proof. Since $(A C) \subset(C B V)$ is dense in $(C B V)$ and therefore in $(B V)$, we see that proving the norm of the functional (5) to be given by (14) is tantamount to proving the theorem. We proceed to consider, then, the functional (5).

Let $\left(S_{g}\right)$ represent the class of step-functions $\left({ }^{17}\right)$ each of which is continuous at each point of discontinuity of $g$. Since the points of continuity of $g$ are dense in $I$, we infer that $\left(S_{g}\right) \subset(B V)$ is dense relative to $(A C)$. Now $\int_{0}^{1} x d g$ exists for $x \varepsilon(A C)+\left(S_{g}\right)$, and we define

$$
F(x)=\int_{0}^{1} x d g \quad \text { for } x \varepsilon(A C)+\left(S_{0}\right) .
$$

Clearly we have $F(x)=f(x)$ for $x \varepsilon(A C)$; and since $\left(S_{g}\right)$ is $\subset(B V N)$, we conclude from Theorem 3 that $F$ is continuous on $(A C)+\left(S_{g}\right)$. Thus we obtain $\left({ }^{18}\right)$

$$
\begin{aligned}
\|f\|_{(A C)} & =\sup _{x \varepsilon(A C),\|x\|=1} \int_{0}^{1} x d g=\sup _{x \varepsilon\left(S_{g}\right),\|x\|=1} \int_{0}^{1} x d g \\
& =\sup _{x \varepsilon\left(S_{g}\right),\|x\| \leqq 1} \int_{0}^{1} x d g=\sup _{x \varepsilon\left(S_{g}\right),\|x\|>0} \int_{0}^{1} x d g /\|x\| .
\end{aligned}
$$

Let $\epsilon$ be any positive number and $y \varepsilon\left(S_{a}\right)$, with $\|y\|=1$, satisfy the inequality

$$
\int_{0}^{1} y d g>\|f\|_{(A C)}-\epsilon
$$

$y$ shall now be regarded as fixed. Let $\left(S_{g}^{\prime}\right)$ be the set of step-functions $x$ defined by the condition $x \varepsilon\left(S_{g}^{\prime}\right)$ if and only if $x$ is continuous at each point $t$ where $y$

(17) It should be clearly understood that by a step-function we mean here a function consisting of a finite number of steps each of length $>0$.

(18) See A, p. 430. We use the notation $\|x\|=(x, \theta)=\int_{0}^{1}|x(t)| d t+T_{0}^{1}(x)$. 
is continuous. Then $\int_{0}^{1} x d g$, for $x \varepsilon\left(S_{g}^{\prime}\right)$ and $\|x\|=1$, is a continuous bounded function of the heights of the steps in $x$ and so assumes a maximum; thus there exists a particular step-function $x_{0} \varepsilon\left(S_{g}^{\prime}\right)$, with $\left\|x_{0}\right\|=1$, such that

$$
\int_{0}^{1} x d g \leqq \int_{0}^{1} x_{0} d g \quad \text { for } x \varepsilon\left(S_{o}^{\prime}\right),\|x\|=1,
$$

which implies

$$
\int_{0}^{1} x d g /\|x\| \leqq \int_{0}^{1} x_{0} d g /\left\|x_{0}\right\| \text { for } x \boldsymbol{\varepsilon}\left(S_{o}^{\prime}\right),\|x\|>0 .
$$

Let $t_{0}$ satisfy the condition

$$
\left|x_{0}(t)\right| \leqq\left|x_{0}\left(t_{0}\right)\right|
$$

for $t \varepsilon I$,

and set

$$
\begin{aligned}
t_{0}^{\prime} & =\inf \underset{t}{E}\left[x_{0}(u)=x_{0}\left(t_{0}\right) \text { for } t<u<t_{0}\right], \\
t_{0}^{\prime \prime} & =\sup \underset{t}{E}\left[x_{0}(u)=x_{0}\left(t_{0}\right) \text { for } t_{0}<u<t\right], \\
J_{0} & =\underset{t}{E}\left[t_{0}^{\prime} \leqq t \leqq t_{0}^{\prime \prime}\right] .
\end{aligned}
$$

Finally, let $x_{\lambda} \varepsilon\left(S_{g}^{\prime}\right)$ be defined thus

$$
x_{\lambda}(t)= \begin{cases}(1+\lambda) x_{0}\left(t_{0}\right) & \text { for } t \varepsilon J_{0}, \\ x_{0}(t) & \text { for } t \varepsilon I-J_{0} .\end{cases}
$$

Then we have

$$
\int_{0}^{1} x_{\lambda} d g=\int_{0}^{1} x_{0} d g+\lambda \cdot x_{0}\left(t_{0}\right) \cdot \Delta_{J_{0}}^{\prime} g
$$

and since $\left|x_{0}(t)\right|$ is actually greater for $t \varepsilon J_{0}$ than it is for $t$ immediately to the left or right of $J_{0}$, there exists an $\eta>0$ such that we have

$$
\left\|x_{\lambda}\right\|=\left\|x_{0}\right\|+\lambda \cdot\left|x_{0}\left(t_{0}\right)\right| \cdot\left|J_{0}\right|+\lambda \cdot\left|x_{0}\left(t_{0}\right)\right| \cdot \nu\left(J_{0}\right) \quad \text { for } \lambda>-\eta .
$$

The function

$$
H(\lambda)=\frac{\int_{0}^{1} x_{\lambda} d g}{\left\|x_{\lambda}\right\|}=\frac{\int_{0}^{1} x_{0} d g+\lambda \cdot\left[x_{0}\left(t_{0}\right) \cdot \Delta_{J_{0}}^{\prime} g\right]}{\left\|x_{0}\right\|+\lambda \cdot\left[\left|x_{0}\left(t_{0}\right)\right| \cdot\left|J_{0}\right|+\left|x_{0}\left(t_{0}\right)\right| \cdot \nu\left(J_{0}\right)\right]}
$$

is of the form $(a+b \lambda) /(c+d \lambda)$ with $c d>0$, and it has a maximum at $\lambda=0$. Hence we have $H^{\prime}(0)=(a d-b c) / c^{2}=0$ and $a / c=b / d$; i.e.,

$$
\int_{0}^{1} x_{0} d g /\left\|x_{0}\right\|_{i}=\left|\Delta_{J_{0}}^{\prime} g\right| /\left[\left|J_{0}\right|+\nu\left(J_{0}\right)\right] \text {. }
$$


Combining our results we obtain

$$
\|f\|_{(A C)}-\epsilon<\int_{0}^{1} y d g \leqq \int_{0}^{1} x_{0} d g=\int_{0}^{1} x_{0} d g /\left\|x_{0}\right\|=\left|\Delta_{J_{0}}^{\prime} g\right| /\left[\left|J_{0}\right|+\nu\left(J_{0}\right)\right] ;
$$

and since $\epsilon$ is an arbitrary positive number, we conclude that $\|f\|_{(A C)}$ is not greater than the number (14).

On the other hand, if $J$ is any closed subinterval of $I$ and the function $x_{J}$ is defined by

$$
x_{J}(t)= \begin{cases}\operatorname{sgn}\left(\Delta_{J}^{\prime} g\right) /[|J|+\nu(J)] & \text { for } t \varepsilon J \\ 0 & \text { for } t \varepsilon I-J\end{cases}
$$

we see at once

$$
x_{J} \varepsilon\left(S_{o}\right), \quad\left\|x_{J}\right\| \leqq 1, \quad \int_{0}^{1} x_{J} d g=\left|\Delta_{J}^{\prime} g\right| /[|J|+\nu(J)],
$$

whence

$$
\begin{aligned}
&\left|\Delta_{J}^{\prime} g\right| /[|J|+\nu(J)] \leqq \sup _{x} \varepsilon\left(S_{g}\right),\|x\| \leqq 1 \\
& \int_{0}^{1} x d g \\
& \sup _{x} \varepsilon\left(S_{g}\right),\|x\|=1 \\
& \int_{0}^{1} x d g=\|f\|_{(A C)} .
\end{aligned}
$$

Thus $\|f\|_{(A C)}$ is not less than the number (14), and the theorem is proofed.

It may be worth while to point out here that the formula (14) provides a good basis for computation. For example, in the case of $g(t)=4 t(1-t)$, the value (14) is assumed for

$$
J=J_{0}=\underset{t}{E}\left[0 \leqq t \leqq 2^{1 / 2}-1\right],
$$

for which $\nu\left(J_{0}\right)=1$, and the norm is $4\left(2^{1 / 2}-1\right)\left(2-2^{1 / 2}\right) / 2^{1 / 2}=.69$ approximately. In the case of $g(0)=g(1)=0, g(.49)=-10, g(.51)=10$ and $g$ linear on each of the closed intervals $[0, .49],[.49, .51],[.51,1]$, the value (14) is assumed for

$$
J=J_{0}=\underset{t}{E}[.49 \leqq t \leqq .51],
$$

for which $\nu\left(J_{0}\right)=2$, and the norm is 20/2.02 =9.90 approximately. Minor variants of this example show that $\|f\|_{(B V)}$ can be arbitrarily close to $|g(1)|+\sup _{t \varepsilon I}|g(t)|$; and other examples to indicate that each of the estimates (12) and (13) is in a sense the best possible can readily be constructed.

We may observe also that the inequality $\left|T_{0}^{1}(x)-T_{0}^{1}(y)\right| \leqq T_{0}^{1}(x-y)$ implies that if $x_{1}$ is an arbitrary point in $(B V)$, we have 


$$
\begin{aligned}
\sup _{x \varepsilon(B V),\left(x, x_{1}\right)>0} \mid f(x) & -f\left(x_{1}\right) \mid /\left(x, x_{1}\right) \\
& \geqq \sup _{x \varepsilon(B V),\left(x, x_{1}\right)>0}\left|f\left(x-x_{1}\right)\right| /\left(x-x_{1}, \theta\right) \geqq\|f\|_{(B V)} ;
\end{aligned}
$$

i.e., that the Lipschitz modulus of a continuous additive functional $f$ at any point in the space is never less than its Lipschitz modulus at the zero-element $\theta$.

5. Weak topologies in $(B V)$. The two forms of functionals (3) and (2) respectively provide the basis for the following

Definitions. $A$ sequence $x_{n}(n=1,2,3, \cdots)$ of elements of $(B V)$ will be said to converge weakly $(S)$ [to converge weakly $(W)$ ] if and only if $\lim _{n \rightarrow \infty} f\left(x_{n}\right)$ exists for every functional $f$ additive and continuous [additive and uniformly continuous] on $(B V)$.

It is clear that convergence of a sequence $x_{n}$ in the metric (1) implies that $x_{n}$ converges weakly $(S)$, and that convergence of $x_{n}$ weakly $(S)$ implies convergence of $x_{n}$ weakly $(W)$. That implications do not hold in the reverse direction may be seen from more or less trivial examples. From (2), which is also the general form of the continuous additive functional on the Banach space $(L)$, as has been remarked in $\S 3$, it is clear that the weak $(W)$ topology of $(B V)$ is equivalent to the topology introduced in $(B V) \subset(L)$ by the weak topology of $(L)$. Since $(B V) \subset(L)$ is strongly dense in $(L)$, it is apparent that the weak closure of $(B V) \subset(L)$ is $(L)$. It has been shown earlier that $(L)$ is weakly complete $\left({ }^{19}\right)$.

THEOREM 7. In the topology of weak $(S)$ convergence, $(B V)$ is complete.

Proof. Let $x_{n}(n=1,2,3, \cdots)$ be any sequence in $(B V)$ satisfying the condition

$$
\lim _{n \rightarrow \infty} \int_{0}^{1} x_{n} d g \text { exists } \quad \text { for every } g \varepsilon(C) ;
$$

and let $\bar{x}_{n}$ be a function associated with $x_{n}(n=1,2,3, \cdots)$ as follows:

$$
\bar{x}_{n}(t)= \begin{cases}\lim _{t_{1 \rightarrow t, t_{1}>t}} x_{n}(t) & \text { for } 0<t<1, \\ 0 & \text { for } t=0, t=1 .\end{cases}
$$

Then we have $\int_{0}^{1} x_{n} d g=\int_{0}^{1} \bar{x}_{n} d g$ for every $g \varepsilon(C)$ and every $n$. For fixed $n$,

$$
\int_{0}^{1} \bar{x}_{n} d g=-\int_{0}^{1} g d \bar{x}_{n}=\int_{0}^{1} g d\left(-\bar{x}_{n}\right) \quad(n=1,2,3, \cdots)
$$

is a continuous additive functional $\left({ }^{20}\right)$ on the Banach space $(C)$, with norm equal to $T_{0}^{1}\left(-\bar{x}_{n}\right)=T_{0}^{1}\left(\bar{x}_{n}\right)$. The condition (15) implies that each $g \varepsilon(C)$ has a

(19) See Banach, loc. cit., pp. 141-142.

${ }^{(20)}$ That is, linear functional, in the sense of Banach, loc. cit. 
bounded sequence of images under the sequence of transformations (16). It follows from a theorem of Banach and Steinhaus $\left({ }^{21}\right)$ that the sequence of norms $T_{0}^{1}\left(\bar{x}_{n}\right)(n=1,2,3, \cdots)$ is bounded. Therefore the sequence of functions $\bar{x}_{n}$ is uniformly bounded, and from a theorem of Helly $\left.{ }^{22}\right)$ we conclude the existence of a subsequence $\bar{x}_{n_{i}}(i=1,2,3, \cdots)$ which converges pointwise for all $t \varepsilon I$ to a function $x_{0} \varepsilon(B V)$. From Lebesgue's convergence theorem we infer that $\bar{x}_{n_{i}}$ converges in the mean to $x_{0}$; and from Theorem 1 above we conclude that $\int_{0}^{1} \bar{x}_{n_{i}} d g$, and therefore $\int_{0}^{1} x_{n} d g$, tends to $\int_{0}^{1} x_{0} d g$ for every $g \varepsilon(C)$.

One may readily verify the following remarks.

(i) In each of the weak topologies, the weak limit of a sequence $x_{n}$ $(n=1,2,3, \cdots)$ in $(B V)$ is not unique in the sense of uniqueness determined by metric equality in $(B V)$; it is, however, unique in the space $(L)$.

(ii) In contrast to the situation in a Banach space $\left({ }^{23}\right)$, boundedness of the sequence $\left\|x_{n}\right\|(n=1,2,3, \cdots)$, where $\left\|x_{n}\right\|=\left(x_{n}, \theta\right)$, is $n o t$ a necessary condition for weak convergence, in either sense, of a sequence $x_{n}$ in $(B V)$; but boundedness of the sequence $\int_{0}^{1}\left|x_{n}(t)\right| d t(n=1,2,3, \cdots)$ is of course necessary.

6. The use of the metric

$$
(x, y)=\int_{0}^{1}|x(t)-y(t)| d t+\left|L_{0}^{1}(x)-L_{0}^{1}(y)\right|
$$

where $L_{0}^{1}(z)$ stands in general for the (Peano) length of the function $z(t)$ on $I$.

When this metric is employed the situation is as described in the following

THEOREM 8. Each uniformly continuous additive functional on $(B V)$ [or on $(C B V)$ or on $(A C)]$ can be expressed in the form (2). The general form of the continuous additive functional on $(B V)$ is (3), on $(C B V)$ is (4), and on $(A C)$ is (8). Conversely, each integral of the kind specified is such a functional on the corresponding space. The functional (8) on $(A C)$ satisfies a Lipschitz condition at any given point $x_{1} \varepsilon(A C)$ if and only if $h(t)$ satisfies a Lipschitz condition on $I$; in this event the integral (8) can be expressed in the form (2), with $g(t)=h(0)$ $-h(t)$, and this integral defines a functional $f$ on $(B V)$ which satisfies a Lipschitz condition on the entire space $(B V)$; and the Lipschitz modulus of $f$ on $(B V)$ [or on $(C B V)$ or on $(A C)]$ is the same at each point $x_{1}$ of $(B V)$ [or of $(C B V)$ or of $(A C)]$ as it is for the entire space, being equal to the Lipschitz modulus of $g(t)=h(0)-h(t)$ on $I$.

We shall endeavor to indicate the proof of these results without going into excessive detail. Naturally it must be noted at the outset that since the metric (17) is not homogeneous (i.e., does not satisfy the condition (ax, ay)

(21) See, for example, Banach, loc. cit., p. 80.

(22) See Helly, Über lineare Funktionaloperationen, Sitzungsberichte der Wiener Akademie, IIa, vol. 121 (1912), p. 283.

(23) See, for example, Banach, loc. cit., p. 133. 
$=|a| \cdot(x, y)$, for $a$ a real number $)$, the spaces considered are neither of the type $(\alpha)$ nor of the type $\left(\alpha^{*}\right)$, so that the results of $\S 2$ of A cannot be drawn upon. In particular, there would seem to be no a priori reason why a uniformly con tinuous additive functional should satisfy a Lipschitz condition $\left({ }^{24}\right)$; that such is the case, however, will presently appear. It is a simple matter to show that a continuous additive functional on $(B V)$ or one of its subspaces here considered is homogeneous $\left({ }^{25}\right)$.

Let $f$ be any additive and uniformly continuous functional on $(B V)$. As in the proof of Theorem 5.2 of $\mathrm{A}$ and of Theorem 2 above, introduce the family of step-functions $\xi_{t}(u)$ and define $f\left(\xi_{t}\right)=g(t)$. In view of the uniform continuity of $f$, let $\delta>0$ be such that

$$
|f(x)-f(y)|<1 \quad \text { for }(x, y) \leqq \delta .
$$

Let $0 \leqq t_{1}<t_{2}<1$ and let $m$ satisfy the condition

$$
m \int_{0}^{1}\left|\xi_{t_{1}}-\xi_{t_{2}}\right| d u=\int_{0}^{1}\left|m \xi_{t_{1}}-m \xi_{t_{2}}\right| d u=\delta .
$$

Then we have

$$
m\left|g\left(t_{1}\right)-g\left(t_{2}\right)\right|=m\left|f\left(\xi_{t_{1}}\right)-f\left(\xi_{t_{2}}\right)\right|=\left|f\left(m \xi_{t_{1}}\right)-f\left(m \xi_{t_{2}}\right)\right|<1,
$$

whence

$$
\left|g\left(t_{1}\right)-g\left(t_{2}\right)\right|<1 / m=\int_{0}^{1}\left|\xi_{t_{1}}-\xi_{t_{2}}\right| d u / \delta=\left|t_{1}-t_{2}\right| / \delta ;
$$

i.e., $g(t)$ satisfies a Lipschitz condition on the interval $0 \leqq t<1$. Consider

$$
x_{1}(t)=\left\{\begin{array}{ll}
0 & \text { for } 0 \leqq t<1, \\
k & \text { for } t=1,
\end{array} \quad x_{2}(t)=\left\{\begin{aligned}
0 & \text { for } 0 \leqq t<1 \\
-k & \text { for } t=1
\end{aligned}\right.\right.
$$

Since $\left(x_{1}, x_{2}\right)=0$ and $\left(x_{1},-x_{2}\right)=0$, we have $f\left(x_{1}\right)=f\left(x_{2}\right)=f\left(-x_{2}\right)=-f\left(x_{2}\right)=0$; and from the additivity of $f$ it follows that the value of $f(x)$ is independent of the value of $x(1)$. The sequence of step-functions $z_{n}(t)$ employed in the proof of Theorem 5.2 of A will then have the property that in the present metric $\left(z_{n}, \bar{x}\right) \rightarrow 0$, with $\bar{x}$ identical with $x$ except at $t=1$ where it differs from $x$ by $1+T_{0}^{1}(x)-L_{0}^{1}(x)$. The argument set forth in that proof then shows that $f(x)$ can be expressed as $\int_{0}^{1} x(t) d g(t)$, where $g(t)$ is Lipschitzian on $I$; i.e., $f(x)$ can be given the form (2), where ess $\sup _{t \varepsilon I}|\alpha(t)|=M$ is the Lipschitz modulus of $g$ on $I$. The existence of a Lipschitz modulus for $f$ on $(B V)$, and its

(24) For example, the linear functionals $f(x)=k x(k \neq 0)$ on the Euclidean space $E_{1}$ metrised with $(x, y)=\left|x^{3}-y^{3}\right|$, which is not homogeneous, do not satisfy a Lipschitz condition.

(25) Compare the reasoning used in the proof of Theorem 2.4 of $\mathrm{A}$ and make use of the relation $\left|L_{0}^{1}(x)-L_{0}^{1}(y)\right| \leqq T_{0}^{1}(x-y)$; see inequality (4) of Adams and Lewy, On convergence in length, Duke Mathematical Journal, vol. 1 (1935), pp. 19-26. 
equality to $M$, then follows from the relation

$$
\begin{aligned}
M & =\sup _{0 \leqq t_{1}<t_{2} \leqq 1}\left|g\left(t_{1}\right)-g\left(t_{2}\right)\right| /\left|t_{1}-t_{2}\right| \\
& =\sup _{0 \leqq t_{1}<t_{2}<1}\left|f\left(\xi_{t_{1}}\right)-f\left(\xi_{t_{2}}\right)\right| /\left|t_{1}-t_{2}\right| \\
& =\sup _{0 \leqq t_{1}<t_{2}<1}\left|f\left(\xi_{t_{1}}\right)-f\left(\xi_{t_{2}}\right)\right| /\left(\xi_{t_{1}}, \xi_{t_{2}}\right) \\
& \leqq \sup _{x, y \varepsilon(B V),(x, y)>0}|f(x)-f(y)| /(x, y) \\
& \leqq \sup _{x, y \varepsilon(B V),(x, y)>0} M \int_{0}^{1}|x-y| d t \\
& \quad \div\left[\int_{0}^{1}|x-y| d t+\left|L_{0}^{1}(x)-L_{0}^{1}(y)\right|\right] \leqq M .
\end{aligned}
$$

Since $(C B V)$ and $(A C)$ are dense in $(B V)$, a functional $f$ additive and uniformly continuous on either subspace can be extended to be uniformly continuous on $(B V)$. As has been done in the proof of Theorem 5.3 of $\mathrm{A}$, one may then show that the extended functional is additive on $(B V)$. Consequently the form of $f$ is determined as asserted.

- That the general form of the continuous additive functional on $(B V)$ is (3) can be shown by essentially the same argument as has been used in the proof of Theorem 3 above.

To determine the form of the continuous additive functional on $(C B V)$, it should be noted that if the set $P$ in Lemma 2 is of measure zero, $x$ is a singular function. According to a theorem of Morse $\left({ }^{26}\right)$, since $x$ is singular, $\left(x_{n}, x\right) \rightarrow 0$ in the metric (1) implies $\left(x_{n}, x\right) \rightarrow 0$ in the metric (17); hence functions $\lambda, \mu$ exist such that in the metric (17), $(\lambda, \mu)$ is $<\delta$ and $\int_{0}^{1} h(t) \lambda^{\prime}(t) d t$ $-\int_{0}^{1} h(t) \mu^{\prime}(t) d t$ is $\geqq k / 2$. Since any non-vacuous perfect set contains such a set with measure zero, only trivial modifications in the proof of Theorem 4 need now be made in order to establish the desired result.

Since in $(A C)_{0}$ convergence in the metric (17) is equivalent $\left.{ }^{27}\right)$ to convergence in the metric $(x, y)=T_{0}^{1}(x-y)$, it follows that the general form of the continuous additive functional on $(A C)_{0}$ is $(6)$ and on $(A C)$ is (8).

The converse statements concerning the uniform continuity of (2) on $(B V)$ and the continuity of (3), (4), and $(8)$ on $(B V),(C B V)$, and $(A C)$ respectively are very easily verified.

The fact that, if $x_{1}$ is an arbitrary point of $(A C)$, the functional (8) on $(A C)$ satisfies a Lipschitz condition at $x_{1}$ only when $h(t)$, and therefore $g(t)=h(0)-h(t)$, satisfies a Lipschitz condition on $I$ is a consequence of

Lemma 3. The functional (8) has the following property when $x_{1}$ is any point of $(A C)$ :

${ }^{(26)}$ See Morse, Convergence in variation and related topics, these Transactions, vol. 41 (1937), pp. 48-83, Theorem 5.2.

(27) The equivalence is a consequence of Theorems 4 and 5 of Adams and Lewy, loc. cit. 


$$
\begin{aligned}
\sup _{x \varepsilon(A C),\left(x, x_{1}\right)>0}\left|f(x)-f\left(x_{1}\right)\right| /\left(x, x_{1}\right) & \\
& \geqq \sup _{0 \leqq t_{1}<t_{2} \leqq 1}\left|h\left(t_{1}\right)-h\left(t_{2}\right)\right| /\left|t_{1}-t_{2}\right| .
\end{aligned}
$$

Proof. In the light of remarks made in the paragraph following (7) it suffices to prove that the left member of $(18)$ is $\geqq|h(b)-h(a)| /(b-a)$, where $0 \leqq a<b \leqq 1$, in four cases: (i) that in which $a=0, b=1$, which is trivially verified by taking $x(t)=x_{1}(t)+1$ for $t \varepsilon I$; (ii) that in which $0<a<b<1$ with $a$ and $b$ points of the Lebesgue set $\left({ }^{28}\right)$ for the function $h$; (iii) that in which $a=0$ and $b, 0<b<1$, is a point of the Lebesgue set for $h$; and (iv) that in which $b=1$ and $a, 0<a<1$, is a point of the Lebesgue set for $h$.

To dispose of case (ii) let $0<\delta<(b-a) / 2$, let $\epsilon$ be an arbitrary number (positive, negative, or zero), and consider the function

$$
x_{\delta, \epsilon}(t)=\left\{\begin{array}{ll}
x_{1}(t) & \text { for } 0 \leqq t \leqq a, b \leqq t \leqq 1 \\
x_{1}(t)+\epsilon(t-a) / \delta & \text { for } a \leqq t \leqq a+\delta \\
x_{1}(t)+\epsilon & \text { for } a+\delta \leqq t \leqq b-\delta, \\
x_{1}(t)-\epsilon(t-b) / \delta & \text { for } b-\delta \leqq t \leqq b .
\end{array} .\right.
$$

For each $\delta$ and $\epsilon$ we have

$$
\begin{aligned}
\int_{0}^{1}\left|x_{\delta, \epsilon}(t)-x_{1}(t)\right| d t= & |\epsilon| \cdot(b-a-\delta), \\
\left|f\left(x_{\delta, \epsilon}\right)-f\left(x_{1}\right)\right|= & \left|f\left(x_{\delta, \epsilon}-x_{1}\right)\right|=\left|\int_{0}^{1} h(t)\left[x_{\delta, \epsilon}^{\prime}(t)-x_{1}^{\prime}(t)\right] d t\right| \\
= & \left|\int_{a}^{a+\delta} h(t) \epsilon / \delta d t-\int_{b-\delta}^{b} h(t) \epsilon / \delta d t\right| \\
= & \mid \epsilon[h(a)-h(b)\rfloor+\epsilon \int_{a}^{a+\delta}[h(t)-h(a)] d t / \delta \\
& \left.-\epsilon \int_{b-\delta}^{b} \mid h(t)-h(b)\right] d t / \delta \mid \\
\geqq & |\epsilon| \cdot|h(b)-h(a)|-|\epsilon| \eta_{1}(\delta)-|\epsilon| \eta_{2}(\delta),
\end{aligned}
$$

where, as $\delta \rightarrow 0$,

$$
\begin{aligned}
& \eta_{1}(\delta)=\int_{a}^{a+\delta}|h(t)-h(a)| d t / \delta \rightarrow 0, \\
& \eta_{2}(\delta)=\int_{b-\delta}^{b}|h(t)-h(b)| d t / \delta \rightarrow 0 .
\end{aligned}
$$

(28) See, for example, Titchmarsh, The Theory of Functions, Oxford, 1932, p. 364. For each $h$ summable on $I$ the "Lebesgue set" of points $t$ where $\int_{t}^{t+\delta}|h(u)-h(t)| d u / \delta$ tends to zero with $\delta$ has measure 1. 
For each fixed $\delta$, the function

$$
\begin{aligned}
\phi(\delta, \epsilon)= & L_{0}^{1}\left(x_{\delta, \epsilon}\right)-L_{0}^{1}\left(x_{1}\right) \\
= & \int_{a}^{a+\delta}\left\{1+\left[x_{1}^{\prime}(t)+\epsilon\right]^{2}\right\}^{1 / 2} d t+\int_{b-\delta}^{b}\left\{1+\left[x_{1}^{\prime}(t)-\epsilon\right]^{2}\right\}^{1 / 2} d t \\
& -L_{a}^{a+\delta}\left(x_{1}\right)-L_{b-\delta}^{b}\left(x_{1}\right)
\end{aligned}
$$

vanishes at $\epsilon=0$, and $\partial \phi / \partial \epsilon$ may easily be seen to exist $\left({ }^{29}\right)$ for each $\epsilon$. If $\partial \phi / \partial \epsilon=0$ at $\epsilon=0$, we have

$$
\frac{\left|f\left(x_{\delta, \epsilon}\right)-f\left(x_{1}\right)\right|}{\left(x_{\delta, \epsilon}, x_{1}\right)} \geqq \frac{|h(b)-h(a)|-\eta_{1}(\delta)-\eta_{2}(\delta)}{b-a-\delta+|[\phi(\delta, \epsilon)-\phi(\delta, 0)] / \epsilon|},
$$

with $[\phi(\delta, \epsilon)-\phi(\delta, 0)] / \epsilon \rightarrow 0$ as $\epsilon \rightarrow 0$. If $\partial \phi / \partial \epsilon \neq 0$ at $\epsilon=0$, there exists a unilateral neighborhood of zero such that for $\epsilon$ in this neighborhood, $L_{0}^{1}\left(x_{\delta, \epsilon}\right)$ is $<L_{0}^{1}\left(x_{1}\right)$. Moreover it is evident that $L_{0}^{1}\left(x_{8, \epsilon}\right)$ is a continuous function of $\epsilon$ which becomes positively infinite as $\epsilon \rightarrow+\infty$ or $\epsilon \rightarrow-\infty$. Hence there exists an $\epsilon$, say $\epsilon_{\delta}$, for which $L_{0}^{1}\left(x_{\delta, \epsilon}\right)=L_{0}^{1}\left(x_{1}\right)$. In fact the inequality $\left({ }^{30}\right)$

$$
\begin{aligned}
L_{a}^{a+\delta}\left(x_{\delta, \epsilon}\right)+L_{b-\delta}^{b}\left(x_{\delta, \epsilon}\right)> & T_{a}^{a+\delta}\left(x_{\delta, \epsilon}\right)+T_{b-\delta}^{b}\left(x_{\delta, \epsilon}\right) \\
\geqq & T_{a}^{a+\delta}\left(x_{\delta, \epsilon}-x_{1}\right)-T_{a}^{a+\delta}\left(x_{1}\right) \\
& +T_{b-\delta}^{b}\left(x_{\delta, \epsilon}-x_{1}\right)-T_{b-\delta}^{b}\left(x_{1}\right) \\
= & 2|\epsilon|-T_{a}^{a+\delta}\left(x_{1}\right)-T_{b-\delta}^{b}\left(x_{1}\right)
\end{aligned}
$$

shows that for $|\epsilon|=L_{a}^{a+\delta}\left(x_{1}\right)+L_{b-\delta}^{b}\left(x_{1}\right), L_{0}^{1}\left(x_{\delta, \epsilon}\right)$ exceeds $L_{0}^{1}\left(x_{1}\right)$; this gives an upper bound for $\left|\epsilon_{\delta}\right|$ and shows incidentally that $\epsilon_{\delta} \rightarrow 0$ with $\delta$. Choosing $\epsilon=\epsilon_{\delta}$, we have

$$
\frac{\left|f\left(x_{\delta, \epsilon}\right)-f\left(x_{1}\right)\right|}{\left(x_{\delta, \epsilon}, x_{1}\right)} \geqq \frac{|h(b)-h(a)|-\eta_{1}(\delta)-\eta_{2}(\delta)}{b-a-\delta} .
$$

The inequality (18) now follows at once from (19), (20), and (21).

Although cases (iii) and (iv) are not formally symmetric, it should suffice to examine one of them. In case (iii), for example, we may define

$$
x_{\delta, \epsilon}(t)= \begin{cases}x_{1}(t) & \text { for } b \leqq t \leqq 1 \\ x_{1}(t)-\epsilon(t-b) / \delta & \text { for } b-\delta \leqq t \leqq b \\ x_{1}(t)+\epsilon & \text { for } 0 \leqq t \leqq b-\delta\end{cases}
$$

and find

(29) See, for example, Hobson, loc. cit., 2d edition, vol. 2, Cambridge, 1926, p. 355.

${ }^{(30)}$ See Adams and Lewy, loc. cit., inequalities (2) and (3). 


$$
\begin{aligned}
\int_{0}^{1}\left|x_{\delta, \epsilon}(t)-x_{1}(t)\right| d t & =|\epsilon| \cdot(b-\delta / 2), \\
\left|f\left(x_{\delta, \epsilon}\right)-f\left(x_{1}\right)\right| & =\left|\epsilon h(0)+\int_{0}^{1} h(t)\left[x_{\delta, \epsilon}^{\prime}(t)-x_{1}^{\prime}(t)\right] d t\right| \\
& =\left|\epsilon h(0)-\int_{b-\delta}^{b} h(t) \epsilon / \delta d t\right| \\
& =\left|\epsilon[h(0)-h(b)]-\epsilon \int_{b-\delta}^{b}[h(t)-h(b)] d t / \delta\right| \\
& \geqq|\epsilon| \cdot|h(b)-h(0)|-|\epsilon| \eta_{2}(\delta),
\end{aligned}
$$

whence we may proceed as before in case (ii).

To complete the proof of the results stated in the theorem, it is sufficient to make the following remark. Let $f$ be a continuous additive functional on $(B V)[(C B V)]$, and let $y_{1}$ be an arbitrary point of $(B V)[(C B V)]$. Set

$$
x_{1}(t)=\int_{0}^{t} y_{1}^{\prime}(u) d u, \quad z_{1}(t)=y_{1}(t)-x_{1}(t), \quad t \varepsilon I,
$$

so that $x_{1}$ is the absolutely continuous part, and $z_{1}$ the singular part, of the function $y_{1}$. Then we have, for $y \varepsilon(B V)[y \varepsilon(C B V)]$,

$$
\begin{aligned}
& \sup _{\left(y, y_{1}\right)>0}\left|f\left(y-y_{1}\right)\right| /\left(y, y_{1}\right) \\
& \geqq \sup _{x \varepsilon(A C),\left(x, x_{1}\right)>0}\left|f\left(x+z_{1}-x_{1}-z_{1}\right)\right| /\left(x+z_{1}, x_{1}+z_{1}\right) \\
& =\sup _{x \varepsilon(A C),\left(x, x_{1}\right)>0}\left|f\left(x-x_{1}\right)\right| /\left(x, x_{1}\right), \\
& \text { since }\left|L_{0}^{1}\left(x+z_{1}\right)-L_{0}^{1}\left(x_{1}+z_{1}\right)\right|=\left|L_{0}^{1}(x)+T_{0}^{1}\left(z_{1}\right)-L_{0}^{1}\left(x_{1}\right)-T_{0}^{1}\left(z_{1}\right)\right| \text {. }
\end{aligned}
$$

Brown UnIVERsity,

Providence, R. I.,

The University of California,

Berkeley, Calif. 